\title{
DIGITAL TRANSFORMATION IN ERASMUS MOBILITY APPLICATION PROCEDURES: TRANSFORMING A PAPER-BASED WORKFLOW INTO AN ONLINE SYSTEM
}

\author{
Oliver Tylšar ${ }^{1}$, David Vavřinec ${ }^{1}$, Abílio Cardoso², Isabel Seruca ${ }^{3}$, Zdeněk \\ Neustupa ${ }^{1}$ \\ ${ }^{1}$ Technical University of Ostrava (CZECH REPUBLIC) \\ ${ }^{2}$ Universidade Portucalense, IJP \& REMIT (PORTUGAL) \\ ${ }^{3}$ Universidade Portucalense, REMIT \& Univ. do Minho, Centro Algoritmi (PORTUGAL)
}

\begin{abstract}
In the process of organising exchange mobility, students and higher education staff members must conclude trilateral Learning Agreements that are the basis for the recognition of academic qualifications obtained abroad. According to the PRIME survey, conducted by the Erasmus Student Network in 2010-11, while Learning Agreements (LA) documents may be considered essential to an adequate organisation of credit mobility, there are several issues that hamper their preparation. In fact, the interlocutors involved are often not clearly defined, the information needed to build the agreements is rarely available online and in up to $75 \%$ of the cases, students need to change their learning agreements after their arrival at the receiving Higher Education Institutions ( $\mathrm{HEI}$ ), usually due to changes in their study plans abroad. Furthermore, as the process is mainly paper-based, it is error prone and time consuming.

This paper describes the project developed by the authors, including two Postgraduate Erasmus students, conducted in the context of Erasmus+ Student Mobility for Placements (SMP) at Portucalense University (UPT). The aim of the project is to develop a web-based software platform to automate the process of submitting applications for UPT students exchange and UPT academic and non-academic staff exchange (STA and STT) within the Erasmus+ programme. The application was termed "ErasmusOnlineLAapp" and addresses the dematerialization of the process of organising exchange mobility, transferring a paper-based workflow, which is reliant on timely postal deliveries for signature purposes, into an online system that will allow students to gain considerable time when preparing their studies abroad and induce a greater degree of flexibility and efficiency when needing to revise the Learning Agreement.
\end{abstract}

Keywords: Digital transformation, paper-based workflow, Erasmus mobility applications.

\section{INTRODUCTION}

Most organisations recognise that digital transformation is key to gaining business advantage in today's rapidly changing marketplace. Digital Transformation can, thus, be seen as a deep and accelerating transformation with regard to processes, activities, competencies and models, in order to take advantage of the changes and opportunities offered by the inclusion of digital technologies into an organization [1].

By embracing digital transformation, organisations in all business sectors can capitalise on new opportunities and optimise existing operations to achieve significant business improvement. To achieve this, there is a need for a two-pronged approach of proactively digitalising paper-based processes and leveraging workflow automation technologies and platforms [2].

In the process of organising exchange mobility, students and higher education staff members must conclude trilateral Learning Agreements that are the basis for the recognition of academic qualifications obtained abroad. According to the PRIME (Problems of Recognition in Making Erasmus) survey, conducted by the Erasmus Student Network in 2010-11 [3], while Learning Agreements (LA) documents may be considered essential to an adequate organisation of credit mobility, there are several issues that hamper their preparation. In fact, the interlocutors involved are often not clearly defined, the information needed to build the agreements is rarely available online and in up to $75 \%$ of the cases, students need to change their learning agreements after their arrival at the receiving Higher Education Institutions (HEI), usually due to changes in their study plans abroad. Furthermore, as the process is mainly paper-based, it is error prone and time consuming. 
This paper describes the project developed by the authors, including two Postgraduate Erasmus students, conducted in the context of Erasmus+ Student Mobility for Placements (SMP) at Portucalense University (UPT). The aim of the project is to develop a web-based software platform, integrated and accessed through the UPT academic portal, to automate the process of submitting applications for UPT students exchange and UPT academic and non-academic staff exchange (STA and STT) within the Erasmus+ programme.

The software application should consider the preparation of all the necessary documents online as well as their update along the application process. For instance, for students' exchange, the application allows the LA to be prepared online by the student, and the connection with the coordinators of the sending and receiving HEls for the update of the document, enabling them to fill their own sections, and further approve and sign the document online.

This process therefore transfers a paper-based workflow, which is reliant on timely postal deliveries for signature purposes, into an online system that will allow students to gain considerable time when preparing their studies abroad and induce a greater degree of flexibility and efficiency when needing to revise the Learning Agreement.

Beyond the dematerialization of the process, it is also envisaged that the online software application will allow HEls to better manage the workflows of the learning agreements, rendering the process of approving and managing these documents more efficient, and thus freeing up time for staff members to work on other activities, while improving the quality of the mobility process.

This paper is structured as follows: Section 2 presents the design and rationale for the architecture of the software solution to be developed. In Section 3 the development of the ErasmusOnlineLAapp application is generally depicted, through the identification of the functional requirements, the presentation of the system modelling, and the interface considered for the two main scenarios of application use. Finally, Section 4 concludes with some considerations on the main challenges addressed, the current stage of the project and future steps to be undertaken.

\section{SOLUTION DESIGN}

\subsection{Envisaging the solution}

A requirement set for the software application was its integration, exchange of information and access through the SIUPT system (UPT web-based information system - on line academic portal), developed in PHP. For the back-end development of the application, the PHP Symphony open-source web framework and a MYSQL database were used, while for the front-end development the Bootstrap framework was used. The advantage of the envisaged solution is that besides its integration with SIUPT, it can be integrated with other HEl's academic portals.

\subsubsection{The SIUPT system}

SIUPT is the UPT academic portal. It is mainly designed for publishing public and internal information about the university, study programmes, students, schedules, etc.

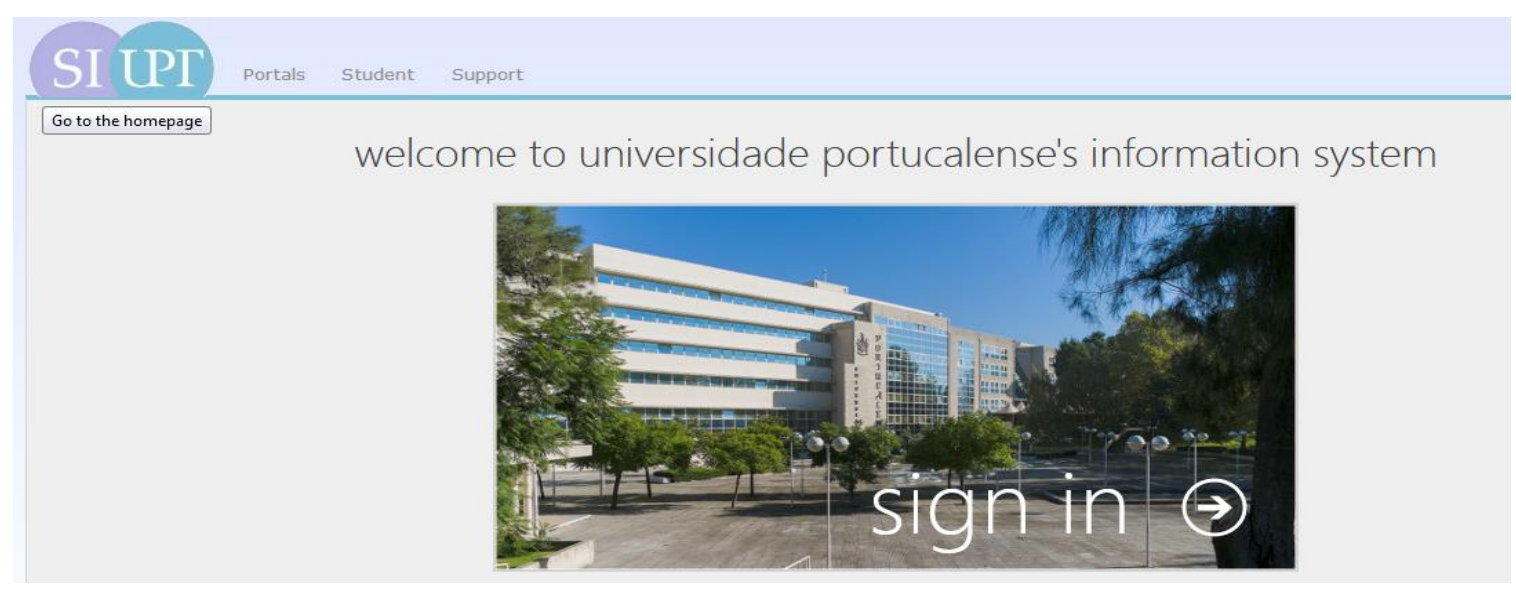

Figure 1 - The SIUPT system 
Access to the SIUPT system is done through an authentication process, allowing a user to login or logout from the portal (Fig. 1). There are three types of user roles (Teacher, Staff, Student), with corresponding access rights. After a user successfully logs in SIUPT, he/she gets relevant information on the portal according to the assigned role.

SIUPT is available in two languages. The main language is Portuguese, while the English version of the portal only offers the most important features and news. In the English version, used within the development of the project, the main menu includes the following options: Portals, Student, Support, Decision (Fig. 2).

\section{Portals}

The option "Portals" redirects the user to other online applications such as the moodle elearning platform. The newly developed application called "ErasmusOnlineLAapp" will be located here. After clicking on the option 'erasmus application' the user will be redirected to the login page of the application.

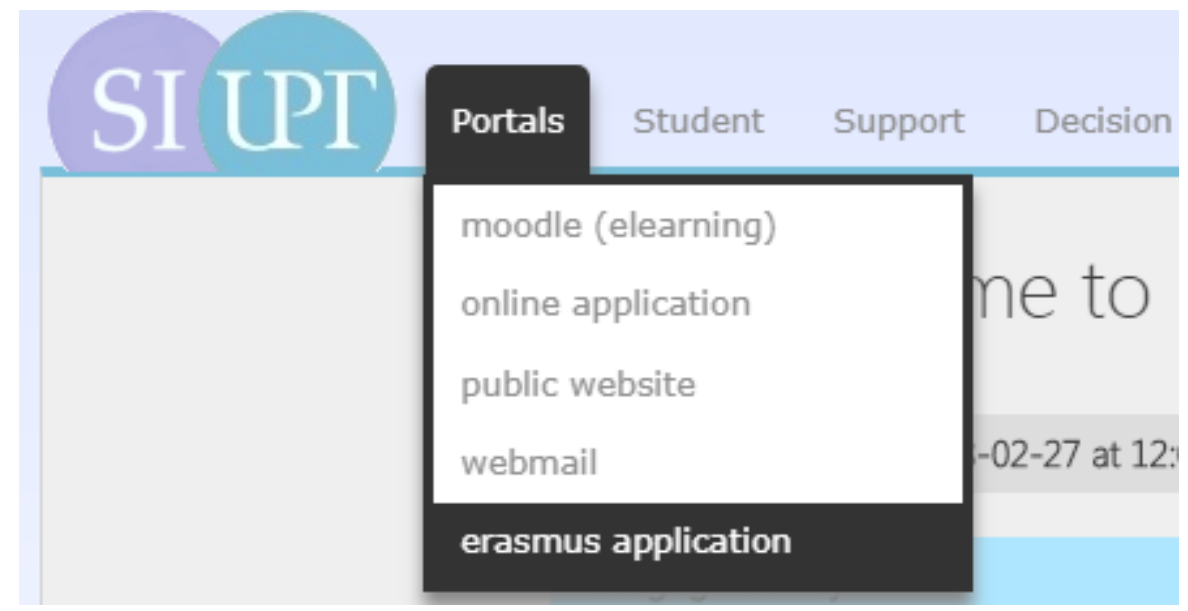

Figure 2 - The SIUPT system main menu - access to ErasmusOnlineLAapp

\section{Student}

This option offers basic information related with students' study purposes (class schedules, evaluation calendars, new enrollment, normal, reset and special period exam enrollment, class enrollment) as well as students' personal information (personal data, enrolled curricular units and grades).

\section{Support}

This section contains useful support information for students, staff and teachers, such as information about helpdesk, restaurant, student cards, etc.

\subsubsection{Information to be transferred}

As a consequence of the integration requirement, the following information was identified to be transferred from the SIUPT system to "ErasmusOnlineLAapp":

- Authorization process (Login; Password)

- Information about users (Students, Staff, Teachers)

- Account type (Student, Staff, Teacher)

- User id number

- First name; Last name; Nationality; Date of birth; Sex (M/F); Email; Phone number

- Study cycle; Academic year; Field of education

- Information about the Sending Institution (UPT) 
- Name; Erasmus code; Address; Country; Faculty/Department; Contact person name, e-mail, phone

- Components (from UPT study programmes): Component code; Component title; Semester; Number of ECTS credits

- Responsible person at the Sending Institution (UPT)

\subsection{Related work}

In the literature review performed, we tried to identify tools and applications that could solve part or the whole process of dematerialization of the paper-based flow related with submitting applications.

Generically there are a set of software tools that address workflow automation, providing online software solutions that automate form based, approval driven workflow and enhance the way information flows between data and systems [4]. While browsing these tools enhanced our insights for envisaging the ErasmusOnlineLAapp interface, all these contributions are more oriented for commercial businesses purposes and do not address the specific issues of the Higher Education Sector.

Although we didn't found any approaches with exactly the same features and addressing the same issues as ErasmusOnlineLAapp, the Online Learning Agreement (OLA) project [5] was considered a useful source of ideas for our development.

The OLA tool has been developed by the European University Foundation and the Erasmus Student Network for the 21st century mobile student. It allows the Learning Agreement to be prepared online, shared with both the home and host universities and be signed using a touchscreen. The platform was developed with the support of Erasmus+ Strategic Partnership project funding (2015-2017) and is now being further upgraded in the second project cycle (2017-2020) by a consortium of university partners and university networks.

In comparison to our application "ErasmusOnlineLAapp", the OLA application has, however, the following shortcomings:

- HEls' information (departments, responsible persons, etc.) are not available to be used within the application;

- Only one option to sign the document is available - drawing interface (missing option to upload a signature as an image);

- Cannot be integrated directly with the SIUPT system, or other similar HEl's academic portals.

\subsection{Target users}

ErasmusOnlineLAapp is intended to be used by students, teachers and staff from UPT. On top of that, it also targets responsible persons of related receiving institutions, involved in the update and signature of the Erasmus application documents.

The application allows the digitalization of the whole process of a student's application for a stay abroad within the Erasmus programme and the connection with the relevant parties for the updating of the document. With the application implemented and integrated in SIUPT, there is no need to scan the LA document multiple times in order to collect all the required signatures. Besides that, the whole process is significantly speeded up and improved, as all the information needed to complete the entire document is available within the application.

\section{ERASMUSONLINELAAPP DEVELOPMENT}

\subsection{Specification and Modelling}

This section contains a summary of the application textual specification targeting the requirements, mostly identified through the study of Erasmus+ documentation for applicants [6], [7] and through the 
information collected in meetings with the UPT International Office staff, as well as the associated modelling of the system.

\section{Textual Specification}

- Login for UPT users (teacher, staff, student) through SIUPT

- Login for receiving institution generated within the application

- Four types of access and rights: Student (UPT - Sending institution); Teacher (UPT - Sending institution); Staff (UPT - Sending institution); Receiving Institution (Generated access to application, not by SIUPT)

- Four types of state for the LA: Editable; Committed; Approved by sending; Approved by both

- Two types of LA: Before mobility; During mobility

- Generated account will be used for the responsible person at the receiving institution to login into application in order to sign and approve the learning agreement

- Access information will be sent to the responsible person of receiving institution just once through an 'Invitation email'; afterwards he will just be notified about new LAs or changes to them

- Sending institution profile (staff, teachers) will be able to manage, sign and approve all LAs committed by students. They can edit, export or remove them.

- Sending institution accounts will have access to the administration section of the application and will be able to manage data about institutions and responsible persons from UPT

- A Receiving institution account will be able to view those LAs where a responsible person's email matches the logged in user's one. This type of account cannot edit LAs except signing and approving them.

- A user will be able to sign the LA using digital drawing or by uploading his signature.

- A student is able to edit a LA until it is committed. Once it's committed, he is no longer eligible to make any additional changes to the LA.

- After the LA is approved by the sending institution, status changes to 'Approved by Sending'.

- After the LA is approved by the receiving institution, status changes to 'Approved by Both'.

- Committed LA can be edited or deleted only by a sending institution account.

- After a LA is 'Approved by Both', student can create a During mobility section of LA.

- A During mobility section containing exceptional changes to the original LA has to be signed by all parties and approved by both institutions once again.

- A student will be able to add components from SIUPT related to his study programme.

- User (student) information will be retrieved from SIUPT.

- Information about the sending institution will be retrieved from SIUPT.

- It will be possible to export a fully approved learning agreement to PDF. 


\section{Modelling of the system}

Fig. 3 shows the Use Case diagram related to the system, while the.relational database model (MySQL) developed for the system is shown in Fig. 4.

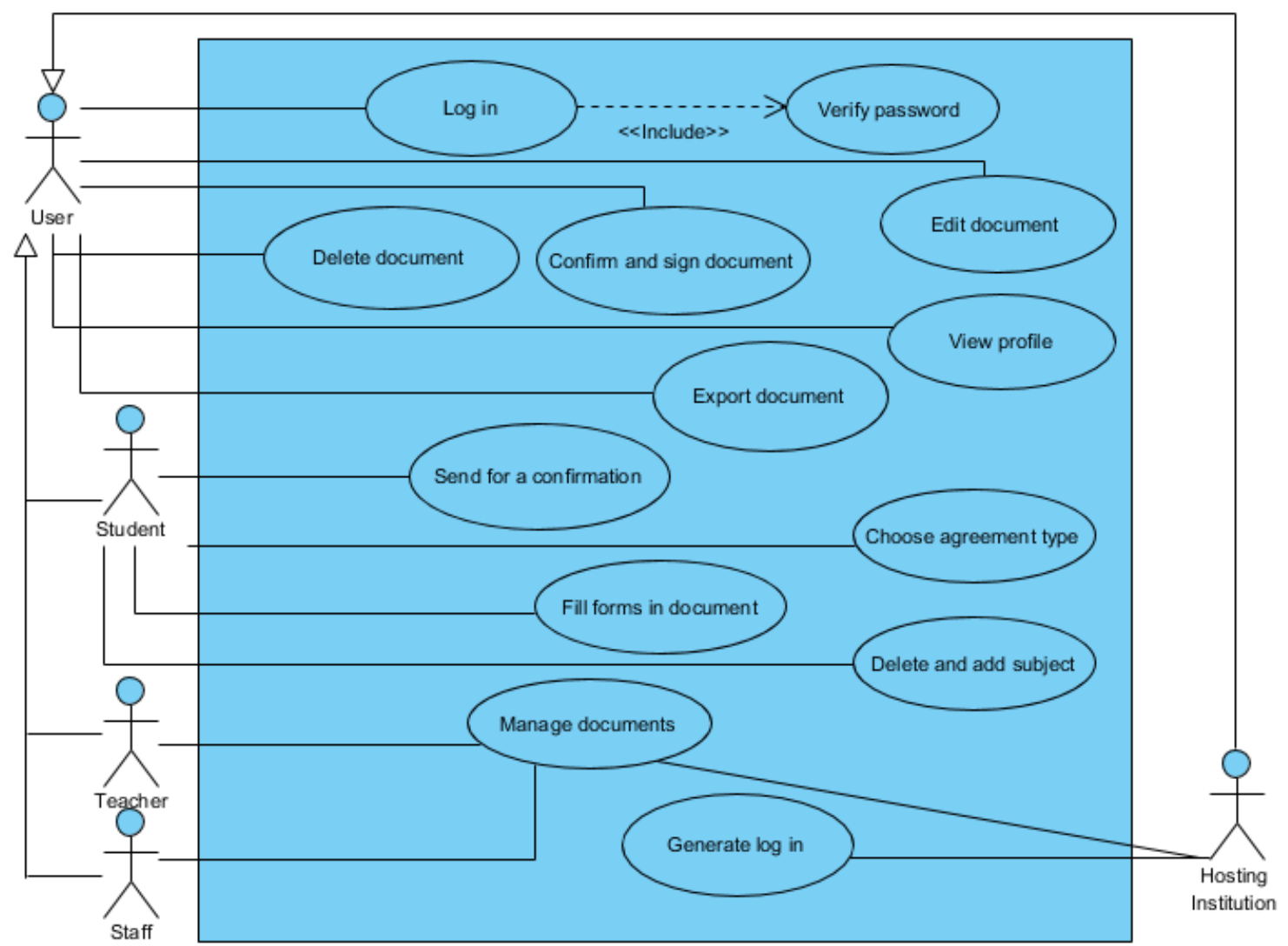

Figure 3 - Use Case Diagram for ErasmusOnlineLAapp

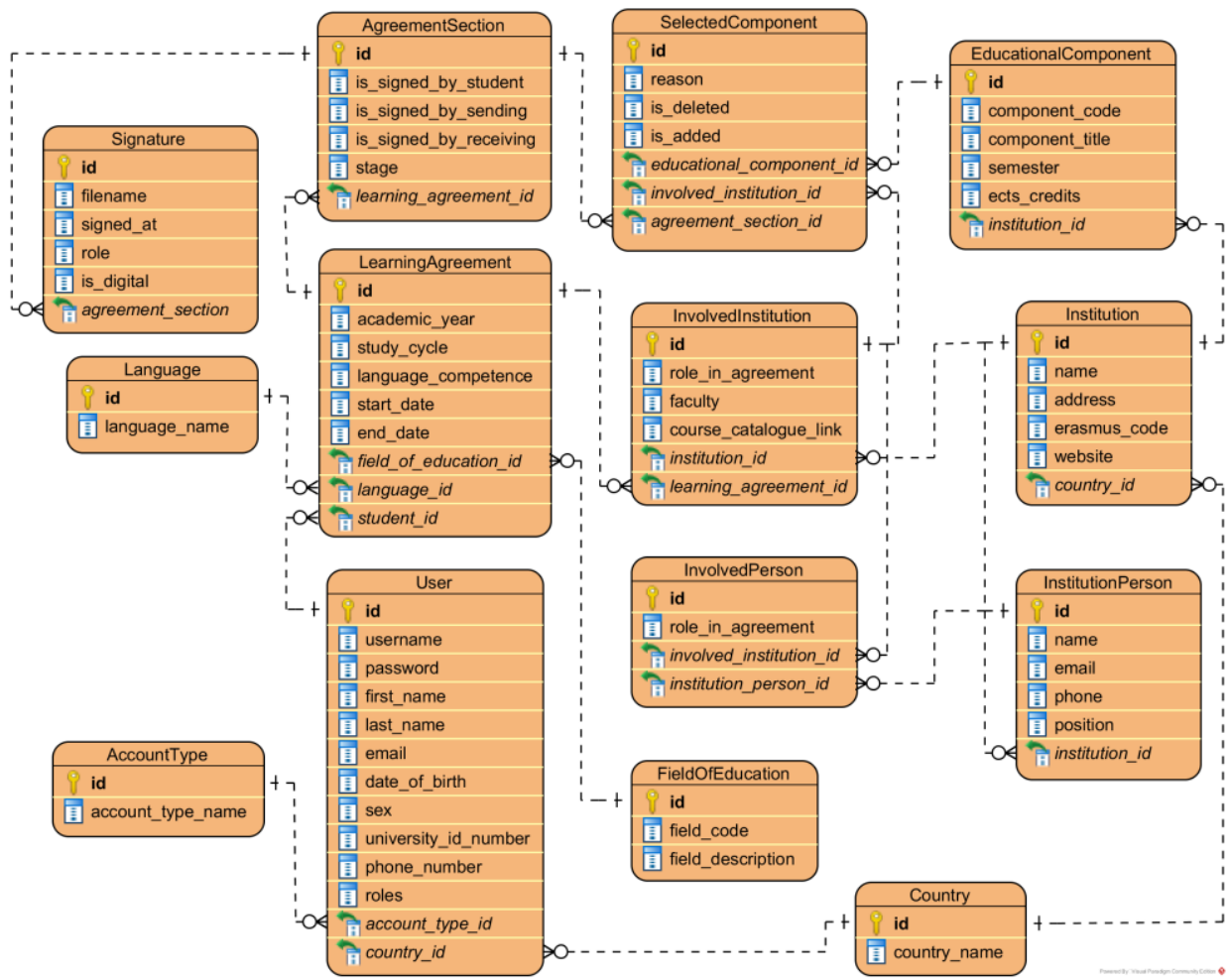

Figure 4 - Database model for ErasmusOnlineLAapp 


\subsection{ErasmusOnlineLAapp Interface}

This section illustrates the two main scenarios of use of ErasmusOnlineLAapp.

\subsubsection{Before the Mobility}

This scenario is concerned with the stage "before the mobility" of the application process, where the student fills the LA document with his personal information, the information about the Sending and Receiving Institutions and the composition (list of components/subjects) of the study programme at the Receiving Institution, as well as the recognition at the Sending Institution of the study abroad, in terms of credits gained and corresponding components. After all this information is gathered, the LA document must be signed by the 3 parties involved (Student, Responsible person at the Sending Institution, Responsible person at the Receiving Institution).

The profiles involved in this stage of the process are listed below together with the description and illustration of the sequence of steps involved using the screens of ErasmusOnlineLAapp.

\section{Student profile:}

- Student creates a Learning Agreement document;

- Student has to fill all the required 6 sections (steps) in LA (Fig. 6);

- If all the sections are fulfilled, student can sign the LA document and commit it;

- Status of LA is then changed to 'Committed' and will show up in the list of LAs that can be seen by profiles with higher access roles (teachers, staff from UPT). From that point, student is no longer entitled to make any further changes;

- After the LA document is signed and approved by both sending and receiving institutions, it is possible to export the LA document to PDF (see LA 46 in Fig. 5);

- Status of LA is then changed to 'Approved by both'.

\section{LEARNING AGREEMENTS}

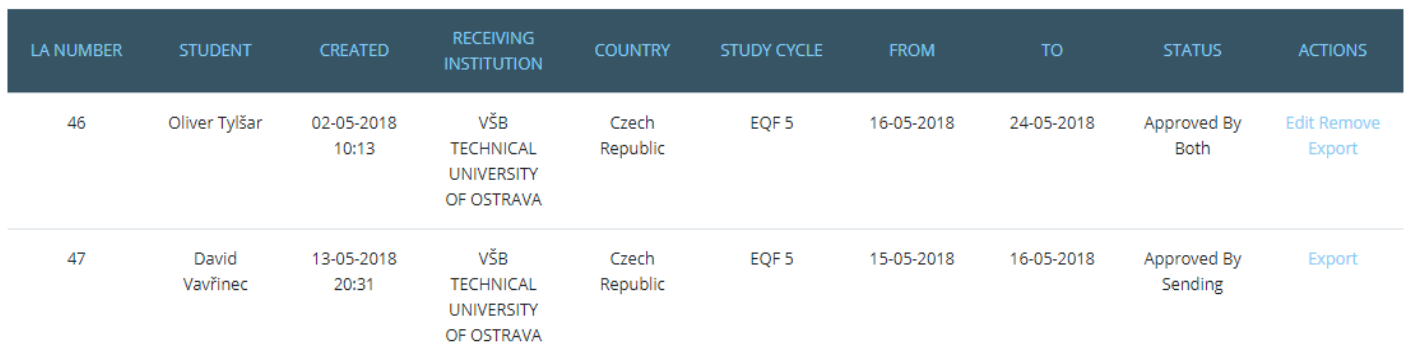

Figure 5 - Before the mobility LA screen

\section{Sending Institution profile (teacher, staff):}

- Sending Institution can manage all the at least committed learning agreements (edit, remove, export);

- Sending Institution can approve LA in section Approval in LA detail;

- LA document can be signed using digital drawing interface or by uploading signature as image;

- After a LA is approved by the Sending Institution, access rights are generated and automatically sent for approval to a responsible person at the Receiving Institution. 


\section{LEARNING AGREEMENT

Figure 6 - Before the mobility LA steps screen

\section{Receiving Institution:}

- As stated before, access for the receiving institution is automatically generated by the application after a LA is approved by the sending institution;

- Access information is sent to the email of the responsible person of a receiving institution;

- The receiving institution can manage all learning agreements. By clicking the button 'View' he can review all the sections of a LA and then sign and approve it in section Approval;

- Signature options stays the same - signature as image or digital signature;

- After approval by the receiving institution, the LA is fully completed and its status is changed to "Approved by both" (LA 46 in Fig. 7).

\section{LEARNING AGREEMENTS}

\begin{tabular}{|c|c|c|c|c|c|c|c|c|c|}
\hline LA NUMBER & STUDENT & CREATED & $\begin{array}{l}\text { RECEIVING } \\
\text { INSTITUTION }\end{array}$ & COUNTRY & STUDY CYCLE & FROM & TO & STATUS & ACTIONS \\
\hline 46 & Oliver Tylšar & $\begin{array}{c}02-05-2018 \\
10: 13\end{array}$ & $\begin{array}{c}\text { VŠB } \\
\text { TECHNICAL } \\
\text { UNIVERSITY } \\
\text { OF OSTRAVA }\end{array}$ & $\begin{array}{l}\text { Czech } \\
\text { Republic }\end{array}$ & EQF 5 & $16-05-2018$ & $24-05-2018$ & $\begin{array}{c}\text { Approved By } \\
\text { Both }\end{array}$ & $\begin{array}{c}\text { Edit Remove } \\
\text { Export }\end{array}$ \\
\hline
\end{tabular}

Figure 7 - Before the mobility detail of LA screen

\subsubsection{During the Mobility}

This scenario is concerned with the stage "during the mobility" of the application process, where the student may change the list of components of his study plan at the Receiving Institution. In that case, for each component change, the student must provide the deleted and the added components, as well as choose an item of a list of options regarding the reason for change. This updated study plan must be approved and signed by both sending and receiving institutions as well as by the student.

The profiles involved in this stage of the process are listed below together with the description and illustration of the sequence of steps involved using the screens of ErasmusOnlineLAapp.

\section{Student profile:}

- Student can create a "LA During mobility" form only if the "LA Before mobility" is "approved by both";

- Student can add or delete components (subjects) previously stated in the "LA Before mobility" (Fig. 8);

- For one "LA Before mobility" form, he can only create one "LA During mobility" form; 
- If any changes are committed by the student, the "LA During mobility" form has to be approved and signed by sending and receiving institutions once again.

\section{Sending institution profile:}

- Sending institution can manage LA_During mobility (edit, remove, export);

- Sending institution can approve LA_During mobility in section "Approval" of the document;

- Signature options are the same - signature as image or digital signature.

\section{LEARNING AGREMENT \\ NUMBER: 46 APPROVED BY BOTH}

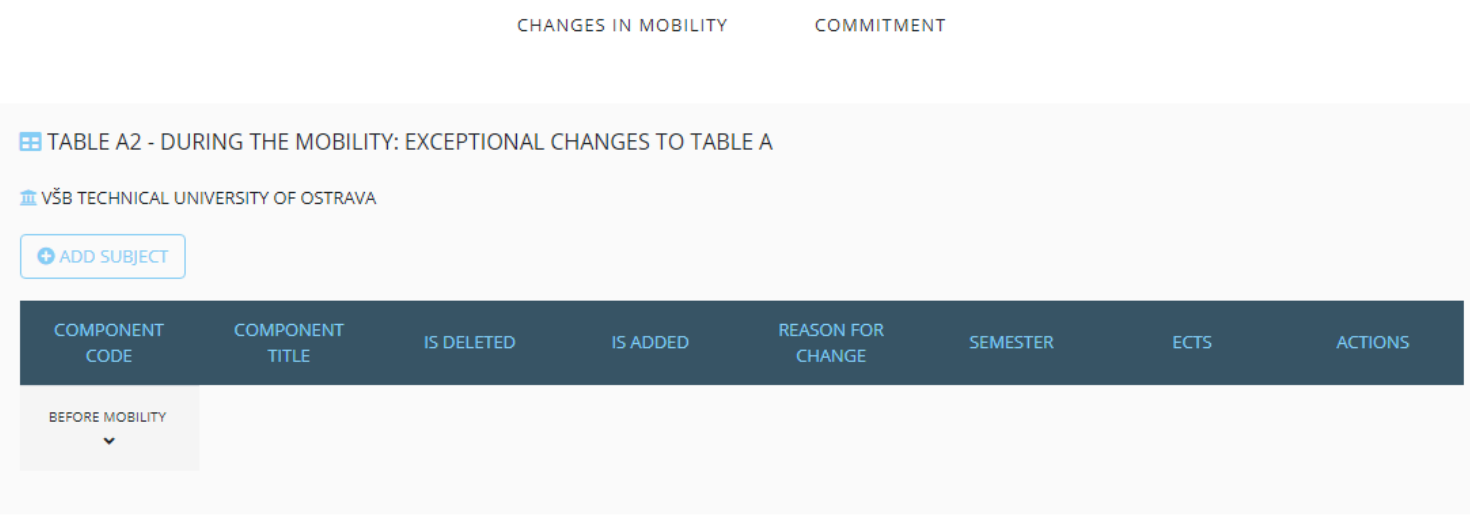

Figure 8 - Editing the During the mobility LA form

\section{Receiving institution:}

- If an account from a receiving institution is related to the LA (responsible person from the receiving institution), it is possible to view the document in the list of Learning Agreements;

- The receiving institution can approve the LA_During mobility in section "Approval" of the document;

- Signature options are the same - signature as image or digital signature.

\section{CONCLUSION AND FURTHER WORK}

As experiences and lessons learned with the development of this project, the following challenges related to the implementation are highlighted:

Analysis of the whole process from the administration point of view - As Erasmus students that already knew how difficult and time consuming is the process of applying to the Erasmus programme, we were excited to take this opportunity to help UPT develop an artefact that would be useful in the near future. However, from our experience, we only knew the application process from a student's point of view. During regular meetings with UPT International Office staff we found out that there is much more to handle in the administration party. Information gained from the meetings were vital for us to understand the whole process from the beginning to the end and were fundamental for both the design of the database and the application user interface, while we did our best to make the later as easy to use as possible.

Actual stage of the implementation of the application - At the final project meeting the application was delivered, which works independently and with its own authentication system. However, the 
application was only tested within a local environment and the integration with the SIUPT system is yet to be finalised as web services of the SIUPT system were under development.

ErasmusOnLineLAapp full integration testing - As stated above, integration with SIUPT is yet under development. In addition, after the software application is installed and running on a university server, it has to be tested properly to make sure that everything works as intended. During the development period, we were testing the application continuously, but only in our local environments. That means that publishing on a university server with possibly a different operating system or database driver, may cause some problems that we haven't experienced before.

Due to time constraints, the support of ErasmusOnLineLAapp for the process of submitting Erasmus applications for UPT academic and non-academic staff exchange (STA and STT) is yet to be developed.

Finally, it is worth noticing that the usage of technologies for the back-end and front-end development of the application, other than the ones used within SIUPT allows ErasmusOnLineLAapp to be integrated with other universities' academic portals as well.

\section{ACKNOWLEDGEMENTS}

We thank Susana Silva and Susana Carvalho from the UPT International Office for their valuable assistance in the project meetings for the identification and requirements validation of ErasmusOnlineLAapp.

\section{REFERENCES}

[1] IDC, "Third platform," 2019, http://www.idc.com/promo/thirdplatform.

[2] Quocirca, "Digital transformation: Bridging the paper and digital gap", 2016. Retrieved from: https://www8.hp.com/h20195/V2/getpdf.aspx/4AA6-5375ENW.pdf.

[3] I“ESN-International Exchange Erasmus Student Network, "PRIME 2010”, Retrieved from: https://www.esn.org/prime/2010

[4] FinancesOnline Reviews for Business, "15 Best Workflow Management Software Solutions", 2019. Retrieved from: https://financesonline.com/top-15-workflow-management-softwaresolutions/

[5] European University Foundation, "Online Learning Agreement+", 2019. Retrieved from: https://www.learning-agreement.eu/start/index.php.

[6] European Comission, "Learning Agreement", 2019. Retrieved from: https://ec.europa.eu/programmes/erasmus-plus/resources/documents/applicants/learningagreement_en

[7] European Comission, "Guidelines on how to use the Learning Agreement for Studies", 2019. Retrieved from: https://ec.europa.eu/programmes/erasmusplus/resources/documents/guidelines-how-use-learning-agreement-studies_en 